SHS Web of Conferences 24, 02005 (2016)

DOI: $10.1051 /$ shsconf/20162402005

C) Owned by the authors, published by EDP Sciences, 2016

\title{
Prediction of BMI by impulsivity, eating behavior and activity level
}

\author{
Xiaxia Jiang \& Yanping Hong \\ College of Education, Hubei University of Science and Technology, Xianning, Hubei, China
}

\begin{abstract}
Objective: Discuss the relationship between the impulsivity, eating behavior and activity level and the body mass index (BMI). Method: Test 147 female college students with the impulsivity questionnaire (BIS-11 and BIS/BAS), Dutch Eating Behavior Questionnaire (DBEQ), Sitting Time Scale (STS) and Exercising Time Scale (ETS). Results: (1) The correlation analysis indicates that BMI and impulsivity $(\mathrm{r}=0.43$ and 0.52$)$ have a significant positive correlation with the sitting time $(\mathrm{r}=0.61)$ and a significant negative correlation with the activity level $(\mathrm{r}=-0.49)$. (2) The path analysis indicates that the reward sensitivity directly affects BMI and indirectly affects BMI through the activity level as well; the eating behavior has an insignificantly direct impact on BMI, because its impact is generated by the intermediary role of induced diet. Conclusion: (1) The impulsivity eating behavior and activity level are closely related to BMI; (2) the activity level, sitting time and induced diet play an intermediary role between the impulsivity and BMI.
\end{abstract}

Keywords: impulsivity; path analysis; eating behavior; BMI

\section{INTRODUCTION}

Among female college students, everyone attaches great importance to weight. Particularly, the weight control behavior of female college students plays a very important role in the daily diet and sports life. The existing studies have found that the weight gain is not only related to eating behavior and exercise habit, but also related to certain personality trait, such as personality trait of impulsivity. Impulsivity generally refers to a kind of tendency for someone who is lack of plan and acts quickly and rarely considers the consequences of such behavior before action ${ }^{[1]}$. At the present stage, there are two orientations for the research of the impulsivity: excitation orientation and inhibition orientation ${ }^{[2]}$. In the excitation orientation, the most important factors are the reward sensitivity and tolerance to the reward delay. In the inhibition orientation, the absence of control ability is the main component of impulsivity. The personality trait of impulsivity is related to the obesity, induced diet, emotional diet, moderate diet and other behaviors. Impulsivity can explain the eating behavior of people with different weight ${ }^{[3]}$.

The relationship between the impulsivity, eating behavior and BMI attracts many scholars to research. The establishment of the model can directly and effec- tively explain the relationship between variables, so it is necessary to establish the model between factors. Davis et al. ${ }^{[4]}$ discussed the intermediary role of the emotional diet in the reward sensitivity and BMI. The result shows that the intermediary role of the emotional diet is not prominent; there is regulation of other more factors between the reward sensitivity and BMI, such as other dietary behavior. Then Davis et al. ${ }^{[5]}$ mainly discussed the relationship between the reward sensitivity, eating behavior, food preference and BMI. The result shows that the reward sensitivity, eating behavior and food preference can truly predict BMI. The path coefficient of food preference for the prediction of BMI is significantly less than that of other paths. Therefore, the author proposes that the popularization of the prediction of BMI by the food preference needs to be further researched. The research of food preference finds that the obese individuals have positive attitude towards healthy and unhealthy food [6]. The obese individuals may not just like eating high-sugar and high-fat food, but like eating something can be eaten. Therefore, the role of food preference in the prediction of BMI is not clear. Therefore, the prediction path of the food preference toward BMI is removed in this research.

The activity level is always an important factor to affect BMI. The correlation between the sitting time 


\section{SHS Web of Conferences}

and inactive physical exercise is significant [7] Meanwhile, Wardle et al. ${ }^{[8]}$ propose that the reward sensitivity individuals are sensitive to food information, but have low sensitivity to physical exercise. Therefore, this research integrates with the activity level and examines the relationship among the impulsivity, activity level and BMI. The research of the prediction of BMI by other side of the compulsivitybehavioral control ability and eating behavior is still very rare. Enticott et al. ${ }^{[9]}$ examined the relationship between self-reported BIS-11 and behavioral control ability of behavior measurement. The result shows that the correlation between BIS-11 and response conflict amount of space Stroop is significant, indicating that BIS-11 may be consistent with the measurement of behavioral control ability.

In order to examine the relationship between the impulsivity, eating behavior and activity level and BMI, this research selects BIS/BAS and BIS-11 questionnaire to measure the reward sensitivity of impulsivity and behavioral control ability, expects to understand the relationship between variables through correlation and path analysis and establishes a prediction model of BMI by the impulsivity, eating behavior and activity level.

\section{RESEARCH OBJECT AND METHOD}

\subsection{Research object}

Among 147 female college students, the average age $\mathrm{M}=21.43 \pm 1.62$. According to the overweight and obese BMI norm of Chinese adults ${ }^{[10]}$, the critical values of the overweight and obese BMI index are respectively 24 and 28 . Among them, when $B M I \geq 24$ 22 , the number of overweight people is 22 ; when $20 \leq \mathrm{BMI}<24$, the number is 98 . The average body mass index (BMI) $\mathrm{M}=22.83 \pm 2.22$.

\subsection{Experimental tools and materials}

\subsubsection{Body Mass Index (BMI)}

Body Mass Index (BMI) respectively measures height and weight by the use of height and weighing machine, keeping two decimal places. BMI is calculated according to the formula of weight $(\mathrm{kg}) /$ height $^{2}\left(\mathrm{~m}^{2}\right)$.

\subsubsection{Sitting Time Scale (STS)}

Sitting Time Scale (STS) is developed by Janssen I et al., which is a scale used to measure the sitting time in life ${ }^{[11]}$. This scale includes six items and five-point score. " 1 " represents "less than or equal to 0.5 hours" while "5" represents "more than five hours". The higher the score is, the longer the sitting time is. This scale has a good reliability and validity. In this research, the coefficient of internal consistency $\mathrm{a}=0.75$.

\subsubsection{Exercising Time Scale (ETS)}

Exercising Time Scale (ETS) is developed by Janssen I, et al, which is a scale used to measure the exercising time in life ${ }^{[11]}$. This scale includes three items and five-point score. "1" represents "less than or equal to 0.5 hours" while " 5 " represents "more than five hours". The higher the score is, the longer the exercising time is. This scale has a good reliability and validity. In this research, the coefficient of internal consistency a $=0.78$.

\subsubsection{BIS/BAS Scale}

BIS/BAS questionnaire is developed by Carver and White ${ }^{[12]}$, which is a questionnaire used to measure impulsivity. First, 20 entries in the English version of the scale are translated into Chinese by psychological professionals; then translate back into English by bilingual psychological professionals (whose mother tongue is Chinese); finally, the Chinese version of BIS/BAS is determined by discussion and added with instructions. This questionnaire includes 20 items, four subscales and four-point score. And BIS questionnaire is used to measure potentially dangerous sensitivity and emotional reaction. Three subscales of BAS are driving force, stimulus seeking and reward reactivity. Among them, the driving force refers to the persistent investment in desired things; the stimulus seeking refers to the investment in potential reward events; the reward reactivity refers to the expectation of the positive emotional investment in reward behavior. As the total score of BAS scale is becoming higher, it indicates that one is more impulsive and more sensitive to reward. As the total score of BIS scale is becoming higher, it indicates that the emotional reaction is stronger. And the scale has a good reliability and validity. In this research, the coefficient of internal consistency of each scale is respectively $0.73,0.80$, 0.76 and 0.79 .

\subsubsection{BIS-11 Scale}

BIS-11 Scale is developed by Patton, Stanford and Barratt and amended by Li Xianyun et al., it is a scale used to measure impulsivity ${ }^{[13]}$. It includes 30 entries, which is divided into six factors: attention factor, exercising factor, self-control factor, cognitive complexity factor, stability factor and cognitive instability factor. According to the frequency of each entry, the record is graded from 1 to 4 ("hardly/never" = 1; "occasionally" $=2$; "often" $=3$; "almost always or always" $=4)$, in which the 11 entries $(4,5,13,14,15,16,17$, $19,20,21,26)$ are reverse scoring. The higher the total score of questionnaire is, the higher the level of individual impulsivity is. And the scale has a good reliability and validity. In this research, the coefficient of internal consistency $\mathrm{a}=0.81$.

2.2.6 Dutch Eating Behavior Questionnaire (DEBQ) Dutch Eating Behavior Questionnaire (DEBQ) is de- 
Table 1. Correlation between the impulsivity, eating behavior, activity level and BMI

\begin{tabular}{|c|c|c|c|c|c|c|c|c|c|c|c|c|c|}
\hline BMI & & & & & & & & & & & & & \\
\hline BIS-2 & $0.43^{* *}$ & & & & & & & & & & & & \\
\hline TBAS & $0.52^{* *}$ & $0.21^{*}$ & & & & & & & & & & & \\
\hline FUNS & 0.08 & 0.14 & $0.54^{* *}$ & & & & & & & & & & \\
\hline DRVE & 0.72 & 0.17 & $0.51^{* *}$ & $0.45^{* *}$ & & & & & & & & & \\
\hline RERE & 0.07 & 0.04 & $0.47^{* *}$ & $0.37^{* *}$ & $0.53^{* *}$ & & & & & & & & \\
\hline BIS & 0.02 & 0.10 & $0.34^{* *}$ & $0.29^{* *}$ & $0.28^{* *}$ & $0.56^{* *}$ & & & & & & & \\
\hline PA & $-0.49^{* *}$ & 0.14 & $0.34^{* *}$ & $0.22^{* *}$ & -0.18 & 0.16 & 0.18 & & & & & & \\
\hline SITT & $0.61^{* *}$ & 0.17 & $-.31^{* *}$ & -0.14 & 0.09 & 0.05 & 0.07 & $-0.80^{* *}$ & & & & & \\
\hline EXET & 0.02 & -0.01 & 0.14 & 0.18 & -.18 & -0.19 & $-0.20^{*}$ & $0.57^{* *}$ & 0.04 & & & & \\
\hline EXT & 0.07 & $0.31^{* *}$ & 0.10 & $-.0 .23^{*}$ & 0.02 & $-026^{*}$ & $-0.26^{* *}$ & 0.01 & 0.02 & 0.05 & & & \\
\hline EMT & 0.16 & $0.23^{* *}$ & 0.03 & 0.16 & -.09 & -0.20 & $-0.24^{* *}$ & 0.01 & 0.13 & 0.18 & $0.41^{* *}$ & & \\
\hline RET & 0.10 & -0.02 & 0.11 & 0.01 & -.01 & 0.05 & 0.04 & 0.05 & 0.07 & 0.18 & 0.18 & $0.23^{*}$ & \\
\hline EAT & 0.07 & $0.30^{* *}$ & 0.12 & $0.21^{*}$ & -.08 & $-0.28^{*}$ & $-0.31^{* *}$ & 0.02 & 0.07 & 0.07 & $0.60^{* *}$ & $0.82^{* *}-0.27^{* *}$ & \\
\hline & BMI & BIS-2 & TBAS & FUNS & DRVE & RERE & BIS & PA & SITT & EXET & EXT & EMT RET & \\
\hline
\end{tabular}

Note: BMI is body mass index; BIS-2 is behavioral control ability; FUNS is stimulus seeking; DRVE is driving force; RERE is reward reactivity; BIS is potential risk sensitivity; PA is activity level, which is exercising time minus sitting time; SITT is sitting time; EXET is exercising time; EXT is induced diet; EMT is emotional diet; RET is restrictive diet; EAT is overall eating behavior, a sum of three kinds of eating behavior.

* represents that $p<0.05 ;{ }^{*}$ represents that $p<0.01$.

veloped by Van Strien et al. ${ }^{[14]}$ which is a questionnaire used to measure eating behavior. First, 33 entries in the English version of the scale are translated into Chinese by psychological professionals and then translated back into English by bilingual psychological professionals (whose mother tongue is Chinese), and then the Chinese version of DEBQ is determined by discussion and added with instructions. This questionnaire includes 33 items, which is divided into three subscales - emotional diet, restrictive diet and induced diet. According to the frequency of each entry, the record is graded from 1 to -5 ("never" $=1$; "occasionally" $=2$; "sometimes" $=3$; "often" $=4$; "always" $=5$ ). As the total score of each subscale is becoming higher, it indicates that the eating behavior is more frequent. The scale has a good reliability and validity. In this research, the coefficients of internal consistency of three subscales are respectively $0.73,0.77$ and 0.79 .

\subsection{Statistical methods}

The statistical analysis is given to all the data through SPSS18.0 statistical software. The main statistical methods are the correlation analysis and path analysis.

\section{RESULTS}

\subsection{Correlation between the impulsivity, eating be- havior, activity level and BMI}

The correlation analysis indicates that BMI has a significant positive correlation with the impulsivity (BIS-2 and TBAS) and sitting time and a significant negative correlation with the activity level. The correlation between various factors is shown in Table 1 .

\subsection{Path analysis of the impact of impulsivity, eating behavior and activity levels on BMI}

In order to carry out in-depth research of the path of the impulsivity through indirect impact of the eating behavior and activity level on BMI, we conduct 6 regression analyses to discuss the impact of various factors on BMI.

Regression analysis 1: With the predictive variables of both sides about the impulsivity, the regression analysis is given to BMI. The prediction of BMI by TBAS and BIS-2 is significant $(\mathrm{F}=3.47, \mathrm{P}<0.001)$.

Regression analysis 2 : With the predictive variable of the impulsivity, the regression analysis is given to eating behavior. The prediction of eating behavior by BIS-2 is also significant $(\mathrm{F}=3.53, \mathrm{P}<0.001)$.

Table 2. Regression of BMI by impulsivity, eating behavior and activity level

\begin{tabular}{llll}
\hline Factor & Beta & $R^{2}$ & $F$ \\
\hline BIS-2 & $0.26^{* *}$ & 0.65 & $13.12^{* *}$ \\
TBAS & $0.44^{* *}$ & & \\
FUNS & $-0.21^{*}$ & & \\
EXE & $-0.20^{*}$ & & \\
SITT & $0.46^{* *}$ & & \\
PA & $0.20^{*}$ & & \\
\hline
\end{tabular}

Regression analysis 3: With the predictive variable of the impulsivity, the regression analysis is given to activity level. The prediction of activity level by TBAS is also significant $(\mathrm{F}=2.25, \mathrm{P}<0.05)$. 


\section{SHS Web of Conferences}

Regression analysis 4: With the predictive variables of the impulsivity, eating behavior and activity level, the regression analysis is given to BMI. The regression equation is significant, as shown in Table 2.

Because the role of prediction of BMI by the activity level and sitting time is significant, the intermediary role of siting time is analyzed. Regression analysis 5: With the predictive variable of the impulsivity, the regression analysis is given to the sitting time. And the regression equation is significant, as shown in Table 3 :

Table 3. Regression of sitting time by impulsivity

\begin{tabular}{llll}
\hline Factor & Beta & $R^{2}$ & $F$ \\
\hline TBAS & $0.33^{*}$ & 0.13 & $2.15^{*}$ \\
\hline
\end{tabular}

Because the role of prediction toward BMI by the eating behavior is not significant while the role of prediction toward BMI by the induced diet is significant, we analyze the intermediary role of induced diet. Regression analysis 6: With the predictive variable of the impulsivity, the regression analysis is given to the induced diet. And the regression equation is significant, as shown in Table 4:

Table 4. Regression of induced diet by impulsivity

\begin{tabular}{llll}
\hline Factor & Beta & $R^{2}$ & $F$ \\
\hline BIS-2 & $0.24^{*}$ & 0.19 & $3.54^{* *}$ \\
\hline
\end{tabular}

In the above multiple regression analysis, the prediction of BMI by impulsivity is significant in regression 1 , and both Beta values are significant ( 0.61 and $0.26)$. In regression 4 , the prediction of BMI by eating behavior is not significant. In regression 2 and regression 3 , the prediction of eating behavior and activity level by impulsivity is significant, and Beta value is also significant $(0.26$ and 0.26$)$. It indicates that the impulsivity can directly affect eating behavior, activity level and BMI, but the eating behavior could not directly affect BMI. The analysis path is shown in Figure 1 (data marked in the Figure are corresponding path coefficients and residual coefficients), and four BMI prediction paths are significant.

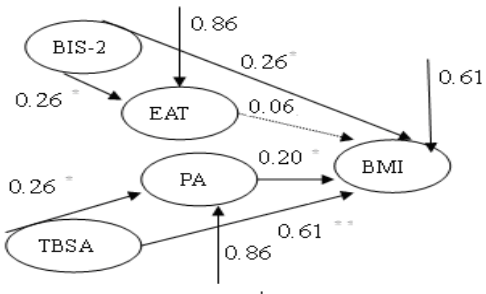

Figure 1. Path analysis of prediction of BMI by impulsivity, eating behavior and activity level

In regression 4, Beta values of the induced diet and sitting time are significant $(0.20$ and 0.46$)$. In regression 5 and 6 , Beta values of the induced diet and sit- ting time are also significant $(0.24$ and 0.33$)$. It indicates that the impulsivity can directly affect BMI and also indirectly affect BMI through the induced eating behavior and sitting time. The analysis path is shown in Figure 2 (data marked in the Figure are corresponding path coefficients and residual coefficients). As shown in Figure 2, six paths that have impacts on $\mathrm{BMI}$ are significant

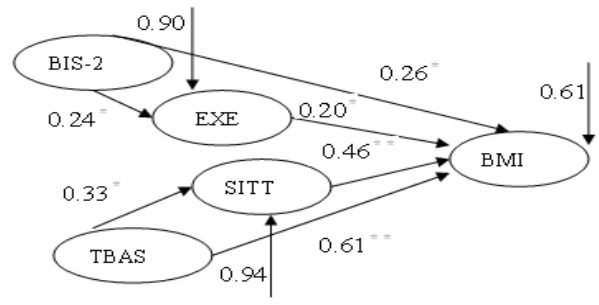

Figure 2. Path analysis of prediction of BMI by impulsivity, external diet and sitting time

\section{DISCUSSION}

4.1 Relationship between the impulsivity, eating behavior and activity level and BMI

This research examines the relationship between two aspects of impulsivity: the reward sensitivity and the relationship among the behavioral control ability, eating behavior, activity level and BMI. The result shows that the impulsivity has a significant correlation with BMI, eating behavior and activity level, indicating that the impulsivity has a basis to explain the diet and activity level of individuals with different weights It is consistent with the research result of $\mathrm{Lu} \mathrm{Yao,} \mathrm{et} \mathrm{al}$ ${ }^{[2]}$. It implies that the personality trait of impulsivity is a risk factor of unhealthy eating behavior and obesity. Compared with individuals with low impulsivity, the individuals with high impulsivity are difficult to resist the temptation of food and prone to take more food.

The score of TBAS has a significant negative correlation with the activity level, indicating that the in dividuals of reward sensitivity reduce the sensitivity to physical exercise. It is consistent with the result of Wardle ${ }^{[8]}$. Therefore, in order to reduce the probability of obesity in women, there is a need to increase their sensitivity toward the benefits of physical exercise and stimulate their interest in physical exercise. It is consistent with a lot of obesity intervention measures. Neumaek-Sztainer et al. ${ }^{[15]}$ and Wang Y et al. ${ }^{[16]}$ increase physical exercise and teach the benefits of physical exercise to improve students' activity level, so as to achieve the goal of weight loss.

\subsection{Intermediary role of activity level, sitting time and induced diet}

The multiple regression analysis shows that the intermediary role of activity level in the reward sensitivity 
and BMI is significant. The reward sensitivity can directly affect BMI indirectly affect BMI through the activity level as well. After adding the activity level, the correlation between the reward sensitivity and BMI is still significant, but the ability to prediction of BMI by the reward sensitivity is increased by $10 \%$. The activity level only plays a part of intermediary role. The more sensitive to the reward is, the lower the activity level is, the less the exercising time is and the greater the BMI is. It indicates that low level of physical exercise is an important factor of overweight.

In regression 4 , the contribution of sitting time in the activity level is greater than the role of exercising time. The result is consistent with the research result of CDC. At the present stage, the exercising time of students is reduced significantly. It is estimated that $20 \%$ of students fail to participate in any extracurricular physical exercise, especially girls. The role of exercising time in BMI is relatively weakened. The sitting time has a significant positive correlation with BMI. It indicates that the more the sitting time is, the greater $\mathrm{BMI}$ is. And it suggests that reduction of the sitting time will be an effective measure to reduce the probability of obesity.

The intermediary role of eating behavior is not significant, which is inconsistent with the result of Davis ${ }^{[6]}$. Perhaps the tested overweight and obese people in this research only account for $15 \%$ and the score of unhealthy eating behavior is not yet super low enough. Therefore, the path between eating behavior and BMI is not significant. Further analysis of eating behavior finds that the intermediary role of induced diet in the behavioral control ability and BMI is significant. It indicates that the behavioral control ability can directly affect BMI and also indirectly affect BMI through the induced diet. The weaker the behavioral control ability is, the more induced eating behavior is and the greater the BMI is. What's more, it is in line with the real life. Due to more availability of high-fat and high-sugar food, food pictures and taste information are filled in our living environment ${ }^{[15]}$. If the behavioral control ability is weaker, it is more prone to be affected by external food information. Even if there is no drive of physical need--"hunger", it can also lead to more eating behaviors. And this model effectively explains the mechanism of impact of induced diet on body weight.

This research tells us that the body weight can be managed by the cognition-behavior intervention model for the intervention of impulsivity ${ }^{[17]}$. The weight loss and obesity prevention can be achieved by changing irrational concept of impulsivity individuals and unhealthy eating behavior. For the intermediary role of induced diet, activity level and sitting time, it suggests that the intervention of the body weight of girls can be started from the eating behavior and activity level. The purpose of weight loss can be achieved by improving the health of eating behavior, stimulating the interest in physical exercise and increasing the physical exercise time. Meanwhile, reduction of the sitting time is also an important measure of body weight control. In the future research, other factors such as the attitude towards the food and physical exercise and the factors about companion and parents ${ }^{[18]}$ can be increased to examine the relationship between many factors and BMI, so as to provide a theoretical basis for the intervention of body weight.

\section{ACKNOWLEDGEMENT}

This paper is financially supported by the Key Provincial (Cultivation) Discipline Pedagogy Funding Project of Hubei University of Science and Technology (No. hkj011).

\section{REFERENCES}

[1] Yeomans MR, Leitch M \& Mobini S. 2008. Impulsivity is associated with the disinhibition but not restraint factor from the Three Factor Eating Questionnaire. Appetite, 50: 469-476.

[2] Lu Yao, He Jinbo \& Zhu Hong, et al. 2015. Impact of different impulsivity traits on adolescent overeating behavior, Chinese Journal of Health Psychology, 23(8): 1229-1232.

[3] Chen Gui. 2013. Research of Relationship between Overweight/Obese Adolescent Delay Discounting and Impulsivity and Eating Behavior. $\mathrm{PhD}$ thesis, Hunan, Second Xiangya Hospital.

[4] Nederkoorn C, Eijs YV \& Jansen A. 2004. Restrained eaters act on impulse. Personality and Individual Differences, 37: 1651-1658.

[5] Davis C, Karen, Robert P \& Levitan et al. 2007. From motivation to behavior: A model of reward sensitivity, overeating, and food preferences in the risk profile for obesity. Appetite, 48: 12-19.

[6] Craeynest M, Crombez G \& Houwer JD, et al. 2005. Explicit and implicit attitudes towards food and physical activity in childhood obesity. Behavior Research and Therapy, 43: 1111-1120.

[7] She Tao. 2015. Correlation analysis of socio-economic factors of China's overweight and obese migrant children, Journal of Guangzhou Institute of Physical Education, 3(35): 35-38

[8] Wardle J, Guthrie C \& Sanderson S, et al. 2001. Food and activity preferences in children of lean and obese parents. International Journal of Obesity, 25: 971-977.

[9] Enticott PG, James RP \& Ogloff JL, et al. 2006. Associations between laboratory measures of executive inhibitory control and self-reported impulsivity. Personality and Individual Differences. 41: 285-294.

10] Working Group of Obesity in China. 2004. BMI reference norm for screening overweight and obesity in Chinese children and adolescents. Chinese Journal of Epidemiology, 25(2): 97-102.

[11] Janssen I, Peter T, \& Katzmarzyk. 2004. Overweight and obesity in Canadian adolescents and their associations with dietary habits and physical activity patterns. Journal of adolescent health, 35: 360-367.

[12] Carver CS \& White TL. 1994. Behavioral inhibition, behavioral activation and affective responses to impend- 


\section{SHS Web of Conferences}

ing reward and punishment: The bis/bas scales. Journal of Personality and Social Psychology, 67: 319-333.

[13] Li Xianyun, Fei Lipeng \& Xu Dong, et al. 2011. Reliability and validity of application of an adapted Chinese version of Barratt Impulsiveness Scale in the community and university population. Chinese Mental Health Journal, 25(8): 610-615.

[14] Van ST. 2002. Dutch Eating Behavior Questionnaire. Manual Thetford, UK: Thames Valley Test Company Limited.

[15] Wei Xiaolu. 2011. Behavioral Impulsivity and Impulse's Influences on Restrained Eating. Master's thesis, Chongqing: Southwest University.
[16] Wang Y, Tussing L \& Young AO. 2006. Obesity prevention in low socioeconomic status urban African-American adolescents: study design and preliminary findings of the HEALTH-KIDS Study. European Journal of Clinical Nutrition, 92-103.

[17] Mobini S, Pearce M \& Grant A et al. 2006. The relationship between cognitive distortions, impulsivity and sensation seeking in a non-clinical population sample. Personality and Individual Differences, 40: 1153-1163.

[18] Chen H, Gao X \& Todd J. 2007. Predictive models for understanding body dissatisfaction among young males and females in China. Behavior Research and Therapy, 45: $1345-1356$ 